\title{
Triterpenos aislados de corteza de Bursera graveolens (Burseraceae) y su actividad biologica
}

\author{
Jorge Robles ${ }^{1 *}$, Ruben Torrenegra ${ }^{1}$, Alexander I. Gray ${ }^{2}$, Catalina Piñeros ${ }^{1}$, Libia Ortiz ${ }^{1}$, \\ Martha Sierra ${ }^{1}$
}

\author{
${ }^{1}$ Grupo de Investigación Fitoquimica Universidad Javeriana GIFUJ, Departamento de Química, Facultad de \\ Ciencias, Universidad Javeriana, Bogotá, Colombia, \\ ${ }^{2}$ Phytochemistry Research Laboratories, Department of Pharmaceutical Sciences, University of Strathclyde, SIBS \\ Building, 27 Taylor Street, Glasgow G4 ONR, Scotland, UK
}

\begin{abstract}
Bursera graveolens (Burseraceae), known in Colombia as "sasafrás", is useful for its medicinal properties and is rich in secondary metabolites. In our research, we carried out antimicrobial tests of several fractions and ethanolic extracts from aerial parts against Bacillus subtilis and Staphylococcus aureus, that showed growth inhibitory activity when applied at 250 $\mathrm{mg} / \mathrm{mL}$ for extracts and $150 \mathrm{mg} / \mathrm{mL}$ for fractions. We carried out an antiinflamatory assay also, that showed $71 \%$ of inhibition by extracts ( $81 \%$ of Indomethacin) and $70 \%$ of inhibition by fractions ( $78 \%$ of Indomethacin). Phytochemical investigation of the bark of Bursera graveolens (Burseraceae) yielded three tetracyclic triterpene acids that have oxygenation in C-3, carboxylic acid in C-21 and unsaturation in $\mathrm{C}-24$ and have been identified as 3-oxotirucalla-8,24-dien-21-oic acid ( $\beta$-elemonic acid), $3 \alpha$-hydroxytirucalla-8,24-dien-21-oic acid ( $\alpha$-elemolic acid) and $3 \alpha$-hydroxytirucalla-7,24dien-21-oic acid. The isolated compounds were identified using spectroscopic methods including one and two-dimensional Nuclear Magnetic Resonance (COSY, HMQC, HMBC, NOESY) experiments and comparison with published data. This is the first report of the isolated compounds in Bursera graveolens and they have a very important chemotaxonomic significance within the Burseraceae family and related families from the order Rutales.
\end{abstract}

Keywords: Bursera graveolens, Burseraceae, antiinflamatory, antimicrobial, triterpenes, tirucallanes.

\section{INTRODUCCION}

Las plantas de la familia Burseraceas se caracterizan por ser árboles o arbustos con sustancias resinosas en casi todos los órganos, presentan canales resiníferos y balsamíferos en el interior de la corteza (Cuatrecasas J. 1957; Swart 1942).

Ocho géneros están representados en América, seis de los cuales son endémicos y entre los que se encuentra el género Bursera, que alberga cuatro especies entre ellas Bursera graveolens que es conocida comúnmente como caraño, sasafrás o tatamaco (Cuatrecasas 1957).

B. graveolens se encuentra distribuida desde Yucatán por Centroamérica hasta Colombia y Perú. La resina producida por esta especie es usada en medicina popular en el tratamiento de hernias, tronchaduras de los pies y para extraer cuerpos extraños de la piel (GarcíaBarriga, 1992). Según Mendieta (1981) los extractos en ron de hojas y cortezas son aplicados localmente para lavar heridas y en forma oral son usados contra el asma, la diarrea, cálculos en el riñón (Bernal et al., 1990).

Según García-Barriga (1992), de esta especie se han realizado algunos estudios fitoquímicos que reportan la presencia de aceites esenciales ricos en monoterpenos y sesquiterpenos, ácido elémico $\left(\mathrm{C}_{35} \mathrm{H}_{45} \mathrm{O}_{4}\right)$, ácido resínico I y II. Bernal; Correa (1990) comenta también la obtención de un compuesto oleánico (ácido 2,3-secoolean-12-en2,3,28-tricarboxilico) y algunos de sus derivados además de una lactona.

En este artículo nosotros describimos el aislamiento y la identificación de tres triterpenos tetraciclicos derivados del esqueleto del tirucalano que se caracterizan por tener una oxidación en el C-3, un ácido carboxílico en el C-21 y una insaturación en el C-24, y que no han sido reportados con anterioridad para esta especie. Además, la presencia de estos triterpenos del tipo tirucalano, mostrando oxidación en el C-21 tienen una importancia quimiotaxonómica, debido a que son los precursores de un grupo de compuestos modificados a partir de ellos que se han aislado de las familias relacionadas a las Burseraceae, mientras estos se han aislados de esta familia (Waterman; Gray, 1987; Liang et al., 1988).

Algunasevaluacionesdeactividadantimicrobiana y antiinflamatoria tanto de los extractos etanólicos como de algunas fracciones de hojas y cortezas de $B$. graveolens, se realizaron basados en algunos reportes que indican la presencia de triterpenos tetraciclicos responsables de la actividad antimicrobiana mostrada por resinas de especies del género Protium y Tetragastris (Gray et al., 
1997). También, se han aislado triterpenos tetraciclicos con marcada actividad antiinflamatoria de la resina cruda de Commiphora mukul como son los compuestos guggulteroles I y III (Chander et al., 1996).

\section{MATERIAL Y MÉTODOS}

Los espectros de RMN fueron realizados en un equipo Bruker AMX-400, utilizando como solvente Cloroformo deuterado en la mayoría de los casos, algunas veces se usó metanol, benceno o piridina deuterados para ayudar a la solubilidad y mejorar la resolución de la señal. Los espectros IR fueron realizados en película seca en un espectrofotometro Perkin-Elmer 781. Los espectros de masas fueron realizados en un espectrómetro de masas JEOL JMS-AX505HA de $70 \mathrm{eV}$.

El material vegetal (hojas y cortezas) de $B$. graveolens fue colectado en el municipio de Agua de Dios (Cundinamarca), a una altura de 400 m.s.n.m. y a una temperatura aproximada de $27^{\circ} \mathrm{C}$. La planta fue identificada en el Herbario Nacional de Colombia y quedó registrada bajo el No. Col. 443597.

La corteza fresca $(2 \mathrm{Kg})$ se sometió a trituración y luego fue extraída por maceración con etanol. El extracto etanólico fue llevado a mínimo volumen en un rotaevaporador de presión reducida y fue fraccionado líquido-líquido con éter de petróleo (14 g), diclorometano (6 g) y acetato de etilo (2.5 g), quedando un residuo etanólico.

Una parte $(7 \mathrm{~g})$ del extracto en éter de petróleo fue sometida a cromatografia en columna empacada con sílica gel $60 \mathrm{G}$ (Merck) y eluída con mezclas de éter de petróleo-acetato de etilo de polaridad creciente. De la fracción 14 obtenidas en proporciones 8:2, precipitó el compuesto (1), un sólido blanco amorfo que se lavó con éter de petróleo, con punto de fusión $122-124^{\circ} \mathrm{C}$; $[\alpha]^{20}{ }_{D}$ $+29.8^{\circ}$ (c $0.0067, \mathrm{Me}_{2} \mathrm{CO}$ ) y que se identificó como ácido 3-oxotirucala-8,24-dien-21-oico.

De la fracción 30 eluída en proporciones 7:3 se separó y purificó un sólido que cristalizó en forma de aguja blancas con metanol, dando un punto de fusión de 210- $212^{\circ} \mathrm{C} ;[\alpha]^{20}{ }_{\mathrm{D}}-20^{\circ}\left(\mathrm{c} 0.003, \mathrm{CHCl}_{3}\right)$ y que se identificó como ácido $3 \alpha$-hidroxitirucala-8,24-dien-21-oico (2).

El ácido $3 \alpha$-hidroxitirucala-7,24-dien-21-oico (3) se purificó de la fracción 38 eluída con proporción 7:3 y precipitó como un sólido blanco amorfo con $\mathrm{CHCl}_{3}$, que dio un punto de fusión de $205-207^{\circ} \mathrm{C} ;[\alpha]^{20}{ }_{\mathrm{D}}-14.9^{\circ}$ (c $0.0073, \mathrm{CHCl}_{3}$ ).

Estos tres compuestos que han sido aislados de otros géneros de la familia Burseraceae, se reportan por primera vez para esta especie.

Para el ensayo antimicrobiano las cepas fueron donadas por el Cepario de bacterias del Laboratorio de Microbiología de la Pontificia Universidad Javeriana y se realizó por el método de perforación en gel (Hostettmann, 1991).

\section{RESULTADOS Y DISCUSION}

Tanto de la fracción en éter de petróleo como de la de diclorometano de corteza de Bursera graveolens, se aislaron por elución en cromatografía en columna, tres triterpenos tetraciclicos (ver parte experimental). Los compuestos aislados fueron identificados como ácido $\beta$-elemónico (1) (Billet et al., 1971; Cotterrel et al., 1970), ácido $\alpha$-elemólico (2) (Tessier et al., 1982; Billet et al., 1971) y ácido 3 $\alpha$-hidroxitirucala-7,24-dien-21oico (3) (Tessier et al., 1982). Estos compuestos fueron identificados por el análisis de sus espectros y comparación de datos espectroscópicos y físicos publicados.

El compuesto 1 (3-oxotirucala-8,24-dien21-oico ó ácido $\beta$-elemónico) presentó en EIMS un ión molecular a m/z 454 que se analizó para $\mathrm{C}_{30} \mathrm{H}_{46} \mathrm{O}_{3}$, indicando 8 insaturaciones equivalentes, el espectro IR mostró bandas intensas a $1724 \mathrm{~cm}^{-1}$ y $1704 \mathrm{~cm}^{-1}$ para $\mathrm{C}=\mathrm{O}$ de cetona y ácido. La función 3-oxo se evidenció de una señal a $\delta$ H 2.53 (ddd) del protón axial H-2 (Liang et al., $1988)$, el cual se acopla con el $\mathrm{H}$ del $\mathrm{C}-1(\delta \mathrm{H} 1.95 / 1.92$, $\delta C$ 35.8). La señal del carbono cuaternario de la función 3-oxo aparece a $\delta \mathrm{C} 218.1$ en el espectro de ${ }^{13} \mathrm{C}$.

$\mathrm{El}$ espectro de $\mathrm{RMN}^{1} \mathrm{H}$ muestra 7 grupo metilo, un protón olefínico a $\delta \mathrm{H} 5.09(\mathrm{t}, J=7.2,14.1)$ que se

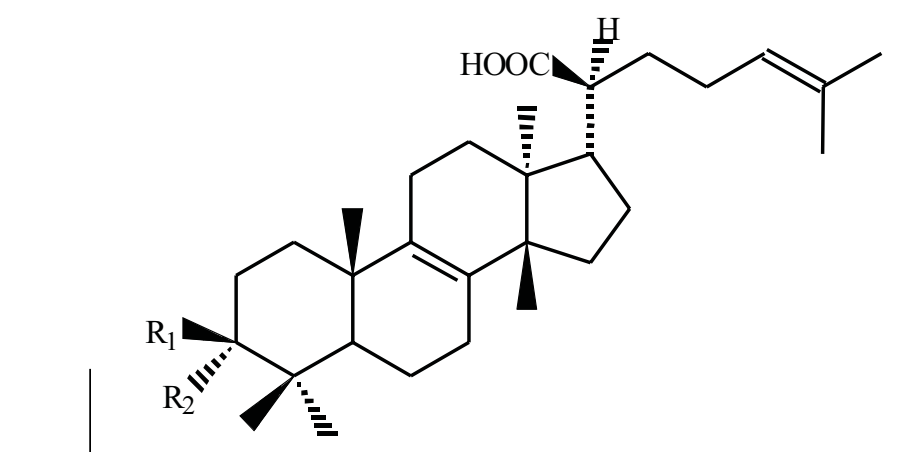

(1) $\mathrm{R}_{1}, \mathrm{R}_{2}=\mathrm{O}$

(2) $\mathrm{R}_{1}=\mathrm{H}, \mathrm{R}_{2}=\mathrm{OH}$

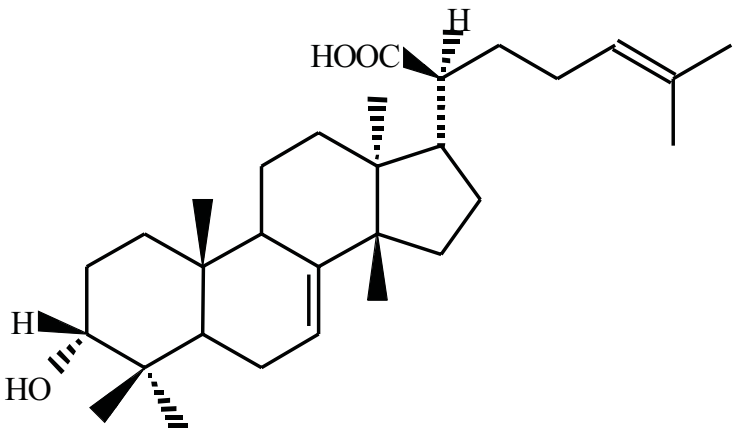

(3) 
acopla directamente con la señal a $\delta \mathrm{C} 123.8 \mathrm{del} \mathrm{C}-24$ y en el espectro COSY está acoplado con los protones de dos metilo a $\delta \mathrm{H} 1.58$ (s, H-26) y a $\delta \mathrm{H} 1.68$ (s, H-27); estos metilos, son geminales y se unen al carbono cuaternario olefínico C-25 como se aprecia en el espectro HMBC. Este acoplamiento también se observó en los compuestos 2 y 3. En este mismo espectro se ve otro par de metilos geminales a $\delta \mathrm{H} 1.05(\mathrm{~s}, \mathrm{H}-29, \delta \mathrm{C} 21.3)$ y a $\delta \mathrm{H} 1.09$ (s, $\mathrm{H}-28, \delta \mathrm{C} 26.8)$ que tienen acoplamiento mutuo a ${ }^{3} J$ con el carbono de $\delta \mathrm{C} 218.3(\mathrm{C}-3, \mathrm{C}=\mathrm{O})$ y con el carbono de $\delta \mathrm{C} 51.7(\mathrm{C}-5, \mathrm{CH})$, también acoplan a ${ }^{2} J$ con el carbono cuaternario de C-4. El C-5 muestra correlación con los protones metilo $\mathrm{H}-19(\delta \mathrm{H} 1.03)$, quienes a su vez se correlacionan con $\mathrm{C}-1$ ( $\delta \mathrm{C} 35.8), \mathrm{C}-10(\delta \mathrm{C} 37.3)$ y con el carbono cuaternario olefínico C-9 ( $\delta \mathrm{C} 132.9)$. El otro lado olefínico, a $\delta \mathrm{C} 134.6$ (C-8) muestra acoplamiento a ${ }^{3} J$ con los protones metilos de $\delta \mathrm{H} 0.90(\mathrm{H}-30)$, los cuales a su vez se acoplan con los carbonos metilénicos

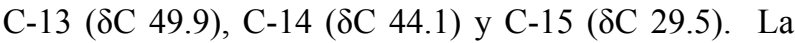
estereoquímica fue deducida a partir del experimento ${ }^{1} \mathrm{H}-$ ${ }^{1} \mathrm{H}$ NOESY donde se aprecia el efecto entre los protones $\mathrm{H}-30(\delta \mathrm{H} 0.90)$ y $\mathrm{H}-17(\delta \mathrm{H} 2.08)$; y entre los protones $\mathrm{H}-18(\delta \mathrm{H} 0.82)$ y $\mathrm{H}-20(\delta \mathrm{H} 2.28)$.

El compuesto 2 (ácido $3 \alpha$-hidroxitirucala8,24-dien-21-oico ó Acido $\alpha$-elemólico) mostró un ión molecular a m/z 456 analizado para $\mathrm{C}_{30} \mathrm{H}_{48} \mathrm{O}_{3}$, dos unidades mas que el compuesto 1 . El espectro de IR muestra bandas de absorción a $3400 \mathrm{~cm}^{-1}$ para un grupo hidroxilo y a 1703 $\mathrm{cm}^{-1}$ para un $\mathrm{C}=\mathrm{O}$ de un ácido. El espectro de $\mathrm{RMN}^{1} \mathrm{H}$ fue muy similar al del compuesto $\mathbf{1}$, pero muestra un protón carbinólico a $\delta \mathrm{H} 3.33(\mathrm{t}, \delta \mathrm{H}-3, J=2.9 \mathrm{~Hz})$ indicativo de un grupo $3 \alpha$-hidroxi (Cotterrell; Wriglesw, 1970; Tessier et al., 1982). El espectro de $\mathrm{RMN}^{13} \mathrm{C}$ fue muy similar al del compuesto $\mathbf{1}$, pero el carbonilo fue reemplazado por un carbono carbinolico que aparece a $\delta \mathrm{C} 76.1$ asignado al C-3. En el HMBC los protones metilos geminales de $\mathrm{H}-28(\delta \mathrm{H} 0.99)$ y H-29 ( $\delta \mathrm{H} 0.79)$ se correlacionan ambos con el C-3 $(\delta \mathrm{C} 76.1)$ y todas las demás correlaciones corresponden con la estructura propuesta (Tessier et al., 1982).

El compuesto 3 (ácido $3 \alpha$-hidroxitirucala7,24-dien-21-oico) muestra un ión molecular de 456 $\mathrm{m} / \mathrm{z}$ analizado para la fórmula $\mathrm{C}_{30} \mathrm{H}_{48} \mathrm{O}_{3}$, igual que el compuesto 2 , sin embargo el espectro de $\mathrm{RMN}^{1} \mathrm{H}$ muestra señales para dos protones olefínicos, uno a $\delta \mathrm{H} 5.25(\mathrm{t}$, $J=7.2,14.7)$ asignado para el $\mathrm{H}-24$ y otro a $\delta \mathrm{H} 5.31$ (d, $J=3.2$ ) asignado al H-7, el cual tiene correlación directa con $\delta \mathrm{C} 119.1$ que no aparece en el compuesto 2. Además, muestra señales para tres protones alifáticos a $\delta \mathrm{H} 2.22$ (m, H-17), a $\delta \mathrm{H} 2.44$ (dt, $J=3.3,11.0, \mathrm{H}-20)$ y a $\delta \mathrm{H} 2.48$ (H-9) que con el carbono de $\delta \mathrm{C} 49.3$ tiene correlación directa.

$\mathrm{El}$ espectro de $\mathrm{RMN}^{13} \mathrm{C}$ muestra siete carbonos cuaternarios (uno menos que el compuesto 2) y siete carbonos terciarios (dos mas que el compuesto 2). La señal de carbono para un metino olefínico a $\delta \mathrm{C} 119.1$ (C7) y un metino alifático a $\delta \mathrm{C} 49.3(\mathrm{C}-9)$ permiten suponer la presencia de un doble enlace entre C-7 y C-8 como lo propuso Tessier et al. (1982).

El aislamiento de varios triterpenos del tipo tirucalano, mostrando oxidación en el C-21, es una evidencia quimiotaxonomica significativa en favor del vínculo existente entre las Burseraceas y sus parientes cercanos, las Meliaceas, Rutaceas y Simaroubaceas del orden Rutales, que presentan compuestos del tipo del Flindisol en los cuales la oxidación del C-21 se une con una oxidación del C-23 y al ciclarse produce un anillo de furano, originando pérdida de 4 carbonos y la formación del grupo de los tetranotriterpenos (limonoides y quasinoides), mientras que en especies de Burseraceas se han aislado triterpenos tetraciclicos derivados del Eufano o Tirucalano como (como los compuestos 1, 2 y 3) y algunos triterpenos pentaciclicos derivados del Oleanano y Ursano (Khalid et al., 1983; Waterman; Gray, 1987).

El extracto etanólico y fracciones de Bursera graveolens presentaron efecto inhibitorio frente $S$. aureus y B. subtilis. La fracción en Acetato de etilo de cortezas de $B$. graveolens mostró mayor actividad frente a $S$. aureus a una concentración de $150 \mathrm{mg} / \mathrm{mL}$. La CMI para el extracto etanólico de hojas frente a $S$. aureus fue de $160 \mathrm{mg} / \mathrm{mL}$, mientras que para el de corteza fue de $190 \mathrm{mg} / \mathrm{mL}$. La CMI del extracto etanolicos de hojas fue de $170 \mathrm{mg} / \mathrm{mL}$ y la de corteza de $180 \mathrm{mg} / \mathrm{mL}$ contra el B. subtilis. Las CMI de las diferentes fracciones fueron de $50 \mathrm{mg} / \mathrm{mL}$. A las concentraciones mencionadas los halos de inhibición presentados por extractos y fracciones fueron similares en diámetro a los presentados por el control positivo que en este caso fue la gentamicina.

Esta acción antimicrobiana parece que se debe a la presencia de compuestos del tipo triterpenos tetraciclicos como los aislados en este estudio, cuya estructura es similar a la del conocido antibiótico ácido fusídico, el cual tiene un núcleo esteroidal y un ácido carboxilico en el C-21 de la cadena lateral, igual a los compuestos aislados 1, 2 y $\mathbf{3}$.

La actividad antiinflamatoria de los extractos etanólicos de $B$. graveolens se evidenció porque el extracto etanólico de corteza a $300 \mathrm{mg} / \mathrm{Kg}$ de peso, dio un $71.66 \%$ de inhibición; mientras, que el extracto etanólico de hojas mostró un $62.0 \%$ de inhibición a la misma concentración. Por su parte, la fracción en éter de petróleo de hojas a $100 \mathrm{mg} / \mathrm{Kg}$ de peso dio un $60.77 \%$ y la fracción de diclorometano en igual concentración dio un $50.48 \%$ de inhibición. La fracción con mayor actividad inhibitoria fue la de éter de petróleo de corteza a $100 \mathrm{mg} / \mathrm{Kg}$ de peso, que mostró un 70.02\% de inhibición al compararse con el antiinflamatorio comercial indometacina $(5 \mathrm{mg} / \mathrm{Kg})$ por el método del edema plantar inducida con carragenina (Cuellar et al., 1997). La actividad se le atribuye a la presencia de ácidos triterpénicos presentes en esta fracción y que fueron aislados de este estudio. Triterpenos tetraciclicos aislados de Burseraceas (Commiphora mukul) parecidos estructuralmente a los aquí obtenidos y conocidos como 
guggulteroles I y III tienen una significativa actividad antiinflamatoria (Chander et al., 1996), igualmente presentan actividad antiinflamatoria compuestos como ácido 3 3,16 $\alpha$-dihidroxilanosta-7,9(11)24-trien-21-oico aislados de Poria cocos (Cuellar et al., 1997).

\section{AGRADECIMIENTOS}

Esta investigación fue financiada por COLCIENCIAS como parte del proyecto "Aislamiento de principios biológicamente activos presente en especies colombianas de la familia Burseraceae", registrado bajo el código No. 1203-05-10122. Los autores están agradecidos con el Herbario Nacional de Colombia por la determinación de la planta. Agradecen a la Pontificia Universidad Javeriana por el apoyo físico en la realización de dicha investigación.

\section{REFERENCIAS}

Billet D, Heitz S, Raulais D, Matschen A 1971. Terpene components of Canarium-boivinii Engl. Phytochemistry 10: 1681-1683.

Bernal H, Correa J 1990. Especies vegetales promisorias de los países del convenio Andrés Bello. Tomo III. Burseracae. Editado por la Secretaria Ejecutivas del Convenio Andrés Bello (SECAB).

Chander R, Khanna AK, Kapoor NK 1996. Lipid lowering activity of guggulsterone from Commiphora mukul. in hyperlipaemic rats. Phytother Res 10: 508-511.

Cotterrell GP, Wriglesw MJ 1970. Chemistry of triterpenes and related compounds. 47. Clarification of nature of tetracyclic triterpene acids of elemi resin. $J$ Chem Soc (C) 739-743.

Cuatrecasas J 1957. Burseraceae. En prima flora Colombiana, 1. Webbia 12: 375-441.

Cuellar MJ, Giner RM, Recio MJ, Just MJ, Mañez S, Rios JL 1997. Effect of the basidiomycete Poria cocos on experimental dermatitis and other inflammatory conditions. Chem Pharm Bull 45: 492-494.

García-Barriga H 1992. Flora medicinal Colombiana. Tomo II. Tercer Mundo editores.

Gray AI, Mitchell AM, Rodriguez JN, De Corredor B, Robles J, Torrenegra R, Cole M D, Cifuentes C 1997. The ups and downs of the ethnobotanical approach to drugs discovery. In: Phytochemical diversity. A source of new industrial products. Special Publication No. 2000 Royal Society of Chemistry. Edited by Stephen Wrigley, Martin Hayes, Robert Thomas and Ewan Chrystal, 74-83.

Hostettmann K 1991. Assay for bioactivity. In: Methods in plants biochemistry. Dey PM and Harborne JB, Eds., Academic Press, London, 6.

Khalid S 1983. Chemistry of the Burseracae. Chapter 10. In: Chemistry and chemical taxonomy of the Rutales. Edited by Waterman and Grundon. Academic Press, London.

Liang GY, Gray AI, Waterman PG. 1988. Tirucallane and oleanane triterpenes from the resin of Aucoumea klaineana. Phytochemistry 27: 2283-2286.

Liang GY, Bates CD, Gray AI, Waterman PG 1988. Chemistry of the Burseraceae .7. The volatile oil of the oleo resin of Aucoumea klaineana collected in Gabon. Planta Med 368-369.

Swart JJ 1942. Flora of Suriname (Netherland Guyana). Vol. III. Part - 2, Edited by The Institute; Printed by JH Bussy, 204-251.

Tessier AM, Delaveau P, Piffault N 1982. Oleoresin of Aucoumea klaineana. 1. Neutral and acid triterpenes. Planta Med 44: 215-217.

Waterman PG, Gray AI. 1987. Chemical systematics. Nat Prod Rep 7: 175-183. 